\title{
Research of High-Speed Circuit Power-Ground Plane Modeling on the Basis of Vector Fitting Algorithm
}

\author{
Lin Qiu, Yunfeng Jia, Honghao Wei \\ School of Electronic and Information Engineering, \\ Beihang University, Beijing, China \\ qiulin@buaa.edu.cn
}

\begin{abstract}
The modeling research is conducted in this thesis, according to the characters of the highway power-ground plane modeling and with the adoption of the vector fitting modeling method. The modeling of a real four-floor PCB power-ground plane is completed, via the comparisons of model time domain, frequency domain response, measured values and simulation values, and the vector fitting model is turned out to be effective and correctly applied in the highspeed circuit power-ground plane modeling.
\end{abstract}

Keywords-High-speed circuit; Vector fitting algorithm; Power-ground plane modeling; Simulation

\section{INTRODUCTION}

The establishment of the equivalent model of powerground plane is the basis of the study on the circuit chip, the basis of signal/power integrity, as well as the precondition of the improvement of the circuit stability. There are a lot of methods for power-ground plane parametric modeling. They have their own advantages and disadvantages. Cavity algorithm is quick and easy but can't use in the irregular shape of the structure. Three-dimensional numerical algorithm has good compatibility, any kinds of circuit models can be calculated. The calculation precision is high, but it takes too much time. Therefore, the study of how quickly and accurately establish the power-ground plane model, the power supply on the basis of carried/signal integrity issues such as research radiation, both for academic research or engineering applications are of great significance.

This thesis introduces the vector fitting algorithm, establishing a power-ground plane mode that is compatible to the time domain circuit solver, with the aim to avoid designers' repeated electromagnetic simulation and correction work and lay the foundation for the establishment of the board electromagnetic compatibility parameterization model.

\section{Modeling Thoughts ON The Basis of Vector FITTING ALGORITHM}

The vector fitting algorithm is a kind of fitting algorithm for rational functions, and it's a fitting method ${ }^{[1]}$. The vector fitting algorithm avoids the equation sickness problems encountered in other value fitting methods, and the simple fraction forms will replace the original term equations ${ }^{[2]}$. Finally the passive networks with the equivalent time domain circuit modes will be substituted into the general SPICE circuit solver for the time domain calculation, to solve the

\author{
Yishuai Zhu \\ Beijing Military Represent Bureau, Army Aviation PLA, \\ Beijing, China \\ plazys@sina.com
}

problems of the slow speed and low efficiency of the electromagnetic-circuit collaborative hybrid simulation. The vector fitting algorithm used in this thesis has the extremely high applicability and matching in the studied power-ground plane modeling methods, which are mainly manifested as the following three points:

(1) Research object of this thesis: Power-ground plane itself is the passive system, which can meet the of vector fitting algorithm's strict requirements of passivity.

(2) A large amount of frequency domain response (parameter S) simulation tests finished in the documents ${ }^{[3]}$, the test data can be directly used in the vector fitting and modeling.

(3) For the advantages and disadvantages of the powerground plane design, the more direct and effective method is to conduct the time domain response. The vector fitting results can directly generate the SPICE netlist, to process the calculations of the time domain responses and the vector fitting model's usability in the power-ground plane design.

The modeling idea of vector fitting algorithm shows as follows:

\section{A. Data Acquisition}

For any passive network, especially the passive linear network, can find one or more rational function to conduct the fitting according to a set of sampling values of the passive network. The basic method is to create a rational function as follow:

$$
R_{m, n}(x)=\frac{P_{m}(x)}{Q_{n}(x)}
$$

In which, $P_{m}(x) \in P_{m}$ and $Q_{n}(x) \in P_{n}$ are the $x^{\prime}$ s m-, norder polynomial. The sick matrix, which may appear in the creation of rational function, can be generally avoided via the vector fitting technologies, to convert the rational function into the pole-residue form, which is shown as the formula (2):

$$
R_{m, n}(s)=\frac{P_{m}(s)}{Q_{n}(s)}=\sum_{i=1}^{n} \frac{\widehat{c_{i}}}{s-p_{i}}+\hat{d}+s \hat{h}
$$


In which, the residue $\hat{c}_{i}$ and pole $p_{i}$ are real numbers or existing in the forms of conjugate complex numbers, and $\hat{d}$ and $\hat{h}$ are real numbers. The vector fitting methods and technologies are tried to solve these residues and poles, to avoid the sick matrix problems in the solutions of the member and denominator coefficients.

\section{B. Circuit Integration}

The vector fitting algorithm can approximate all kinds of parameters, which include but are not limited to: the network's parameter $\mathrm{S}$, admittance parameter $\mathrm{Y}$ or impedance parameter $\mathrm{Z}$, etc, the port parameter analytical expression can be finally obtained at the Laplace domain (domain s). The vector fitting method have the vector fitting results of the single-port circuit's impedance $\mathrm{Z}$ will extend each term into the form of continued fraction, according to each single real pole and the conjugate complex pole subterm, like the formula (3):

$$
\begin{gathered}
Z(s)=d+s e+\sum_{l=1}^{N_{s}} \frac{c_{l}^{s}}{s-a_{l}^{s}}+\sum_{l=1}^{N_{c}}\left(\frac{c_{l}^{c}}{s-a_{l}^{c}}+\frac{{\overline{c_{l}}}^{c}}{s-{\overline{a_{l}}}^{c}}\right) \\
=R_{0}+s L_{\infty}+\sum_{l=1}^{N_{s}} \frac{1}{C_{l}^{s} s+G_{l}^{s}}+\sum_{l=1}^{N_{c}} \frac{1}{C_{l}^{c} s+G_{l}^{c}+\frac{1}{L_{l}^{c} s+R_{l}^{c}}}
\end{gathered}
$$

The same calculation is conducted for the vector fitting results of the single port network's admittance parameter Y. The formula can be obtained (4):

$$
\begin{gathered}
Y(s)=d+s e+\sum_{l=1}^{N_{s}} \frac{c_{l}^{s}}{s-a_{l}^{s}}+\sum_{l=1}^{N_{c}}\left(\frac{c_{l}^{c}}{s-a_{l}^{c}}+\frac{\bar{c}_{l}^{c}}{s-\overline{a_{l}}}\right) \\
=G_{0}+s C_{\infty}+\sum_{l=1}^{N_{s}} \frac{1}{L_{l}^{s} s+R_{l}^{s}}+\sum_{l=1}^{N_{c}} \frac{1}{L_{l}^{c} s+R_{l}^{c}+\frac{1}{C_{l}^{c} s+G_{l}^{c}}}
\end{gathered}
$$

For the formula (3), the first and second terms are the resistances and inductance series branches, the third term is the corresponding series branch of the real number single pole and the fourth term is the corresponding series branch of the conjugate complex pole; for the formula (4), the first and the second terms are the resistance and inductance parallel branches, the third term is the corresponding parallel branch of the real number single pole, the fourth term is the corresponding series branch of the conjugate complex pole. The circuit forms after the vector fitting integration are shown in the Figure 1, in which (a) represents resistance model and (b) represents the admittance model.

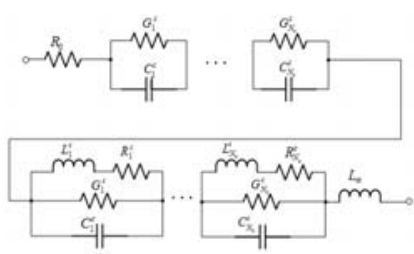

(a) resistance model

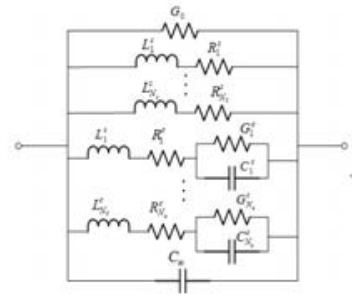

(b) admittance model
Figure 1. Single Port Circuit Models after Integration

Observing the above formula, you can easily find that there are the coupling relations among the values of the components R, L, G and C in the series and parallel branches, and there exist the following relations in the poles and residues:

$$
\begin{aligned}
& \left\{\begin{array}{l}
R_{0}\left(G_{0}\right)=d \\
L_{\infty}\left(C_{\infty}\right)=e
\end{array}\right. \\
& \left\{\begin{array}{l}
C_{l}^{s}\left(L_{l}^{s}\right)=\frac{1}{c_{l}^{s}} \\
G_{l}^{s}\left(R_{l}^{s}\right)=-\frac{a_{l}^{s}}{c_{l}^{s}}
\end{array} \quad l=1,2, \cdots N_{s}\right. \\
& \left\{\begin{array}{l}
\mathrm{C}_{l}^{c}\left(\mathrm{~L}_{l}^{c}\right)=\frac{1}{2 c_{l}^{r}} \\
\mathrm{G}_{l}^{c}\left(\mathrm{R}_{l}^{c}\right)=\frac{-a_{l}^{r} c_{l}^{r}+a_{l}^{i} c_{l}^{i}}{2 c_{l}^{r^{2}}} \\
\mathrm{~L}_{l}^{c}\left(\mathrm{C}_{l}^{c}\right)=\frac{2 c_{l}^{r^{3}}}{\left(c_{l}^{r^{2}}+c_{l}^{i^{2}}\right) a_{l}^{i^{2}}} \\
\mathrm{R}_{l}^{c}\left(\mathrm{G}_{l}^{c}\right)=\frac{-2 c_{l}^{r^{2}}\left(a_{l}^{r} c_{l}^{r}+a_{l}^{i} c_{l}^{i}\right)}{\left(c_{l}^{r^{2}}+c_{l}^{i^{2}}\right) a_{l}^{i^{2}}}
\end{array}\right.
\end{aligned}
$$

In which $\left\{\begin{array}{l}\mathrm{c}_{l}^{c}=\mathrm{c}_{l}^{r}+j \mathrm{c}_{l}^{i} \\ \mathrm{a}_{l}^{c}=\mathrm{a}_{l}^{r}+j \mathrm{a}_{l}^{i}\end{array} \quad l=1,2 \cdots N_{c}\right.$

This thesis mainly focuses on the single port network at the two sides of power-ground plane segmentation of the highway circuit. Therefore, for the data of the single port's parameter $\mathrm{S}$, they can be converted into the parameter $\mathrm{Z}$ via the following formula.

$$
Z=\frac{\left(1+S_{11}\right) \times Z_{0}}{1-S_{11}}
$$


According to the formula (5) - (8), any components' value can be calculated. If the extra constraints are implemented in the vector fitting solution procedure, to ensure $R, L, G$ and $C$ to be positive values, the generated single port circuit model will also be physically realizable, to realize the equivalence of circuit networks to the device ports' characters.

\section{HighWAy CirCUIT POWER-GROUND PLANE MODELING}

A power-ground plane PCB with segmentation structure is created. Firstly, according to the above-said stacking design, a four-floor PCB stacking structure is established. And the PCB's metallic layers consist of the top signal layer, ground layer, power layer and bottom signal layer, and each metal layer is filled with FR4 insulating base material. The top signal layer's wiring thickness and width are $1.7 \mathrm{mil}$ and 10mil respectively. The thickness of the first layer, the FR4 media layer, is $5 \mathrm{mil}$, and the metallic thickness of the ground layer is $1.7 \mathrm{mil}$. The space between the metal layer and power layer are filled with the 15mil-thick FR4 insulating base material. The thickness of power layer metal is also $1.7 \mathrm{mil}$. The bottom signal layer is symmetric to the top signal layer, which means the $1.7 \mathrm{mil}$ wiring thickness and $10 \mathrm{mil}$ wiring width are adopted. The space between the bottom signal layer and power layer is filled with 15mil FR4 insulating material. A $35 \mathrm{~mm}$ long and $2 \mathrm{~mm}$ wide segmentation structure is built at the bottom signal layer. The area has not been covered with copper and the two terminals of the signal lines are connected from the SMA ports, to ensure the vector network analyzer can conduct the test easily. The final domain is shown as Figure 2, real objects as Figure 3.

The measurement of the parameter $S$ at the port for the real object is conducted. The used measuring device is Agilent vector network analyzer, with $0-5 \mathrm{GHz}$ as the test frequency. And the measuring results are shown as Figure 4.

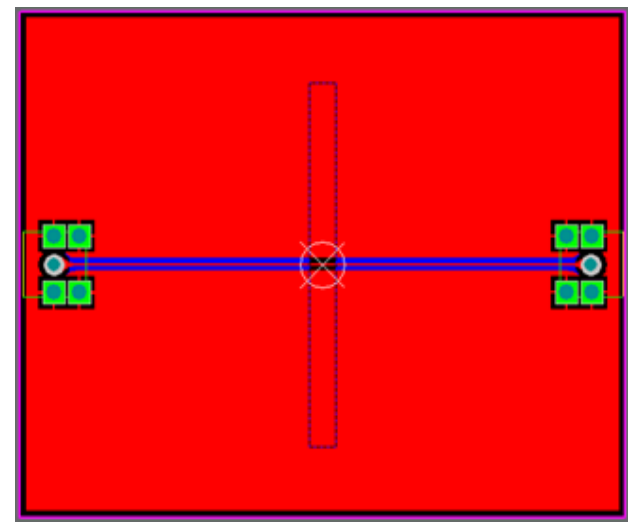

Figure 2. Design for Ground Layer with Segmentation Structure's $4^{\text {th }}$ Layer PCB Domain

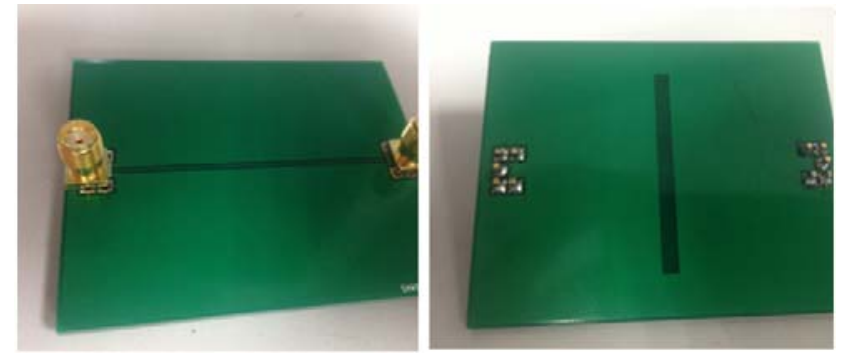

Figure 3. Design for Ground Layer with Segmentation Structure's $4^{\text {th }}$ Layer PCB Domain

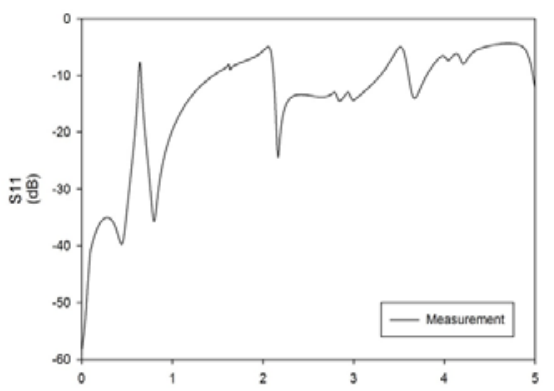

Figure 4. S11 Return Loss Measuring Results (Original Date Used to Conduct The Vector Fitting Modeling)

This thesis utilizes the vector fitting method to conduct the $0-5 \mathrm{GHz}$ frequency band modeling for the highway signal network with the ground layer segmentation structure. The basis of the modeling is the measuring results of the parameter $\mathrm{S}$ of the network. The measuring results' parameter $S$ data are stored in the .txt format, whose unit is $\mathrm{dB}$. As all the data are the calculation results of parameter $\mathrm{S}$, the simulating calculation results adopt the 500 frequency sweeps, which are conducted by the FEM algorithm. Therefore the specific input variation can be defined as a $K \times 1$ dimension $(K=500)$ complex frequency vector s and a $p \times p \times K$ dimension complex data array $\overline{\bar{Y}}_{R A W}$, in which $\mathbf{s}(k)=j 2 \pi f_{k} \quad, \quad \overline{\bar{Y}}_{R A W}(i, j, k)=\left.\overline{\bar{Y}}_{R A W}(i, j)\right|_{f=f_{k}}$, $k=1,2, \ldots N+1$. $K$ is the frequency domain data length, selected after the pretreatment. $p$ is the number of the ports at the built power-ground plane. The measuring method is the dual port measurement, so the port number is 2 . In the finally built model, which is based vector fitting method, is the dual equivalent model. From the comparison expression $\overline{\bar{Y}}=\overline{\bar{k}}^{-1}(\overline{\bar{I}}-\overline{\bar{S}})(\overline{\bar{I}}+\overline{\bar{S}})^{-1} \bar{k}^{-1} \quad\left(\overline{\bar{k}}^{-1}\right.$ is the diagonal matrix's mean square root of the return loss at Port 1 , obtained by the full-wave simulation), the parameter $\mathrm{S}$ and 13 grades of poleresidue model for the fitting can be obtained. And the obtained results are shown as Figure 5. By comparing the vector fitting results and simulating calculation results, the 13-grade vector fitting model can well approach the return loss characteristic parameters of the ground plane S11 with the $35 \mathrm{~mm}$ long and $2 \mathrm{~mm}$ wide segmentation structure. 

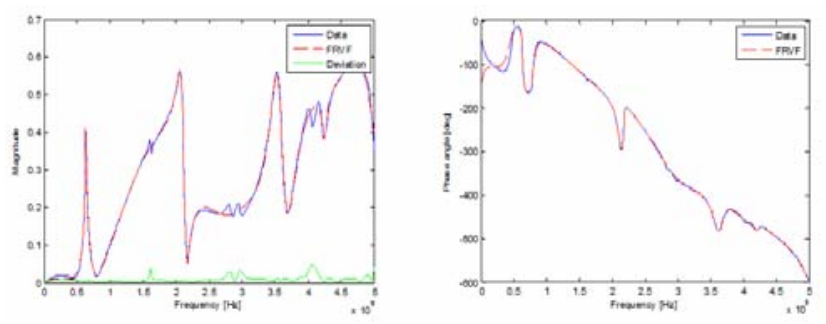

Figure 5. S11 Parameter of the signal two port network Utilizing the Signal Two Port Network S11 Parameter of Ground Layer with Segmentation Structure, Obtained from Vector Fitting (Range and Phase)

\section{CONCLUSION}

This thesis firstly introduces vector fitting modeling method into the highway circuit power-ground plane modeling, to create a PCB of a power-ground plane with segmentation structure according to the modeling thought. Seen from the comparisons of the model's time domain, frequency domain and real-measuring value and simulating value, the return loss characteristic data are obtained from the good approach test in the full frequency band via the 13 grade vector fitting algorithm. This research result verifies the effectiveness and accuracy of the vector fitting method in the power-ground plane modeling.

\section{REFERENCES}

[1] Archambeault B. and Connor S. The effect of decoupling capacitor distance on printed circuit boards using both frequency and time domain analysis. IEEE International Symposium on Electromagnetic Compatibility. 2005, 650-654.

[2] Sievenpiper D., Zhang L. and Broas R. F. J., et al. High-impedance electromagnetic surfaces with a forbidden frequency band. IEEE Transactions on Microwave Theory and Techniques. 1999, Nov., 47(11): 2059-2073.

[3] Jia Yunfeng, Li Zi-ning, Zhang Guangyu, Wu Liang. Co-Simulation of PCB and Enclosure to Predict Radiated Emission of Avionic Equipment. CSAE 2013, IEEE. 